\title{
Spectroscopic Survey of H $\alpha$ Emission Line Stars Associated with Bright Rimmed Clouds
}

\author{
Kensuke Hosoya1 ${ }^{*}$, Yoichi Itoh ${ }^{1}$, Yumiko Oasa ${ }^{2}$, Ranjan Gupta ${ }^{3}$, Asoke Kumar Sen ${ }^{4}$ \\ ${ }^{1}$ Nishi-Harima Astronomical Observatory, Center for Astronomy, University of Hyogo, Kobe, Japan \\ ${ }^{2}$ Faculty of Education, Saitama University, Saitama, Japan \\ ${ }^{3}$ Inter University Center for Astronomy and Astrophysics (IUCAA), Pune, India \\ ${ }^{4}$ Department of Physics, Assam University, Silchar, India \\ Email: *yitoh@nhao.jp
}

How to cite this paper: Hosoya, K., Itoh, Y., Oasa, Y., Gupta, R. and Sen, A.K. (2019) Spectroscopic Survey of $\mathrm{H} \alpha$ Emission Line Stars Associated with Bright Rimmed Clouds. International Journal of Astronomy and Astrophysics, 9, 154-171. https://doi.org/10.4236/ijaa.2019.92012

Received: May 12, 2019

Accepted: June 18, 2019

Published: June 21, 2019

Copyright (c) 2019 by author(s) and Scientific Research Publishing Inc. This work is licensed under the Creative Commons Attribution International License (CC BY 4.0)

http://creativecommons.org/licenses/by/4.0/ Open Access

\begin{abstract}
The results of a spectroscopic survey of $\mathrm{H} \alpha$ emission line stars associated with fourteen bright rimmed clouds are presented. Slit-less optical spectroscopy was carried out with the Inter University Centre for Astronomy and Astrophysics (IUCAA) $2 \mathrm{~m}$ telescope and IUCAA Faint Object Spectrograph and Camera (IFOSC). $\mathrm{H} \alpha$ emission line was detected from 173 objects. Among them 85 objects have a strong $\mathrm{H} \alpha$ emission line with its equivalent width larger than $10 \AA$. Those are classical T Tauri stars. 52 objects have a weak $\mathrm{H} \alpha$ emission line with its equivalent width less than $10 \AA$ and do not show intrinsic near-infrared excess. Those are weak-line $\mathrm{T}$ Tauri stars. On the other hand, 36 objects have a weak $\mathrm{H} \alpha$ emission line $(<10 \AA)$, although they show intrinsic near-infrared excess. Such objects are not common in low-mass star forming regions. Those are misfits of the general concept on formation process of a low-mass star, in which it evolves from a classical T Tauri star to a weak-line $\mathrm{T}$ Tauri star. Those might be weak-line $\mathrm{T}$ Tauri stars with a flared disk in which gas is heated by ultraviolet radiation from a nearby early-type star. Alternatively, we propose pre-transitional disk objects as their evolutional stage.
\end{abstract}

\section{Keywords}

Star Formation, Pre-Main Sequence Stars, T Tauri Stars

\section{Introduction}

A T Tauri star (TTS) is a low-mass star in the pre-main sequence stage. It was first identified as a variable star [1]. Successive studies on low-mass star forming regions, such as the Taurus molecular cloud, revealed two types of TTSs, namely 
classical TTSs (CTTSs) and weak-line TTSs (WTTSs). CTTSs show the H $\alpha$ emission line with its equivalent width larger than $10 \AA$. WTTSs also show the $\mathrm{H} \alpha$ emission line, but its equivalent width is less than $10 \AA$. [2] investigated near-infrared colors of TTSs in the Taurus molecular cloud. They estimated the amount of the interstellar extinction from $E(R-I)$ color and then calculated the dereddened colors of the TTSs. They noted that the WTTSs have the dereddened colors similar to those of normal main sequence stars. On the other hand, the dereddened colors of CTTSs occupy a narrow range in a $(J-H)-(H-K)$ color-color diagram. The range is well fitted by a linear line with an intersection with the reddening vector from an M6 dwarf at $A_{\mathrm{V}}=1.9$ mag (hereafter termed as the dereddened CTTS line). Dereddened colors of approximately half of the CTTSs are redder than that intersection point. Such CTTSs have an intrinsic near-infrared excess. Those are brighter in the $H$ - and/or $K$ band than the radiation expected only from the photosphere. The intrinsic near-infrared excess of a TTS is caused by an optically thick circumstellar disk, and the amount of the excess depends on the optical thickness of the disk, geometric structure of the disk, and viewing angle of the disk [3] [4]. Approximately half of CTTSs show the intrinsic near-infrared excess while the other CTTSs do not. On the other hand, WTTSs do not show the intrinsic near-infrared excess.

A low-mass star evolves from a protostar to a CTTS and subsequently a WTTS. [5] investigated the ages of TTSs in the Taurus molecular cloud. They used Hipparcos parallaxes as well as photometric and spectroscopic information for 72 TTSs. They plotted them on the HR diagram and then estimated their ages by comparing them to a pre-main sequence evolutionary track. It is revealed that the ages of the CTTSs are younger than those of the WTTSs. A solar mass star evolves from a CTTS to a WTTS at the age of $4 \times 10^{6} \mathrm{yr}$. [6] deduced the surface gravity of TTSs in the Taurus molecular cloud from high resolution spectroscopy and then estimated their ages by plotting them on the HR diagram. The fraction of the objects with the intrinsic near-infrared excess decreases with the age. They concluded that the dissipation timescale of the circumstellar disk is $3-4 \times 10^{6} \mathrm{yr}$. The general concept that a low-mass star evolves from a CTTS to a WTTS is well established by observational studies of nearby low-mass star forming regions.

Bright rimmed clouds (BRCs) are molecular clouds harboring an IRAS source and are located at periphery of an HII region. Ultra-violet radiation from OB stars ionizes molecular cloud materials through shock and excites hydrogen atoms at the surface of the cloud, making diffuse $\mathrm{H} \alpha$ emission. [7] and [8] presented comprehensive catalogs of BRCs, in which 89 BRCs are listed. Properties of the clouds and young stellar objects (YSOs) associated with the clouds have been extensively investigated. [9] carried out millimeter mapping observations of molecular clouds associated with the W5 HII region. They noticed that molecular clouds facing the HII region show a steep density gradient toward the HII region due to the compression of the HII region to the clouds. [10] conducted 
$\mathrm{H} \alpha$ grism spectroscopy and optical narrow-band imaging observations of 28 BRCs. They detected $460 \mathrm{H} \alpha$ emission line stars. [11] carried out wide-field near-infrared imaging observations of 32 BRCs. They identified 2099 objects as YSO candidates. These observational studies confirmed the formation of low-mass stars in BRCs. [12] carried out a near-infrared survey of 44 BRCs. They indicated that stars with bluer colors are located closer to the OB star and those with redder colors were closer to the IRAS source. [13] presented a near-infrared image of BRC 14. They revealed that the fraction of YSO candidates to all sources, the extinction of all sources, and the near-infrared excess of the YSO candidates increased from the outside of the rim to the center of the molecular cloud. These results indicated that star formation proceeds from outside to the center of the cloud.

We conducted slit-less optical spectroscopy of 14 BRCs to identify $\mathrm{H} \alpha$ emission line stars. We discuss evolution process of a low-mass star in a massive-star forming region based on the relationship between the $\mathrm{H} \alpha$ emission line and its intrinsic near-infrared excess.

\section{Observations and Data Reduction}

Spectroscopic survey of $\mathrm{H} \alpha$ emission line stars were carried out on six nights of 2011 January and 2012 January with IFOSC (IUCAA Faint Optical Spectrograph and Camera) mounted on the IUCAA 2-m telescope at Giravali near Pune, India. We used the IFOSC5 grism and the wide $\mathrm{H} \alpha$ filter, and we did not use any slits. IFOSC has a $2048 \times 2048$ CCD with the field of view of $10.5^{\prime} \times 10.5^{\prime}$. We had spectra centered at $6563 \AA$ with a width of $80 \AA$ and spectral resolution of $9 \AA$. We also obtained $V$-band images. The targets were 14 BRCs listed in [7] and [8] that were observable on the observing date (Table 1). Three frames of 300-s exposure each were obtained for spectroscopy and one frame of $60 \mathrm{~s}$ was obtained for imaging. The OB star making the HII region and the bright rim is located in the observing fields of view for BRCs 15, 24, and 25. However, these OB stars are so bright that their spectra were saturated. The general seeing conditions varied between 0.9 " and 2.0 ".

The object frames were calibrated with the Image Reduction and Analysis Facility (IRAF). Data were processed in standard manner, namely bias subtraction and flat fielding with the twilight frames. We detected point sources in the $V$-band image with SExtractor program. The limiting magnitude is approximately 19 mag. Based on the coordinate of the source, a spectrum image of each object was extracted from the spectral frame. The spectrum extends along a line. We average the counts of each line and the average count was then subtracted from each line of the image. In the process, the continuum flux of the object was subtracted and the emission line appeared as a point source. We detected the emission line via the SExtractor program. We also confirmed the emission line by eye inspection. For the image with the emission line, we extracted a 1-D spectrum from the image prior to the continuum subtraction. An equivalent width of 
Table 1. Properties of the observed bright rimmed clouds.

\begin{tabular}{ccccccc}
\hline Cloud & $\begin{array}{c}\text { RA } \\
\text { [J2000] }\end{array}$ & $\begin{array}{c}\text { DEC } \\
{[\mathrm{J} 2000]}\end{array}$ & HII region & $\begin{array}{c}\text { Spectral type } \\
\text { of the exciting star }\end{array}$ & $\begin{array}{c}\text { Distance } \\
\text { [pc] }\end{array}$ & obs. fields \\
\hline BRC 15 & 05 23 30.1 & +331154 & S 236 & O5V, O6V & 3600 & 6 \\
BRC 16 & 05 19 48.9 & -055205 & S 276 & O7V, O8.5II, O9.5I & 400 & 2 \\
BRC 17 & 05 31 28.1 & +120624 & S 264 & O5V & 450 & 3 \\
BRC 18 & 05 4429.8 & +090854 & S 264 & O5V & 450 & 4 \\
BRC 19 & 053430.7 & -023812 & S 277 & O9.5V & 400 & 1 \\
BRC 21 & 053941.3 & -033712 & S 277 & O9.5V & 400 & 1 \\
BRC 23 & 062258.7 & +230958 & S 249 & O9V & 1900 & 1 \\
BRC 24 & 063455.4 & +042514 & S 275 & O5V & 1400 & 9 \\
BRC 25 & 064103.3 & +102159 & S 273 & O7V & 913 & 3 \\
BRC 26 & 070347.2 & -114547 & S 296 & O7III & 990 & 2 \\
BRC 27 & 070358.7 & -112319 & S 296 & B0V & 990 & 2 \\
BRC 28 & 070443.4 & -102159 & S 296 & B0IV & 990 & 2 \\
BRC 29 & 070452.4 & -120926 & S 296 & O7.5V & 990 & 1 \\
BRC 45 & 071823.7 & -220613 & RCW 14 & O8V & 1930 & 2 \\
\hline
\end{tabular}

the emission line was measured with the IRAF splot task. We did not fit a Gaussian profile. The minimum equivalent width of the detected $\mathrm{H} \alpha$ emission line was $0.3 \AA$.

\section{Results}

Emission lines of $\mathrm{H} \alpha$ were identified from 173 objects (Table 2). Their equivalent widths range from $0.3 \AA$ to $132 \AA$. We investigated near-infrared properties of the emission line stars by using 2 MASS photometries. The near-infrared color-color diagram of the emission line stars are presented in Figure 1. We defined the line parallel to the reddening vector through an M6 dwarf color as the near-infrared excess border. Among the emission line stars, 77 objects are plotted redward of the border. We identified that such objects have the intrinsic near-infrared excess. The color-color diagram of TTSs in the BRCs is significantly different from that of TTSs in Taurus. TTSs with the equivalent width less than $10 \AA$ in Taurus are plotted blueward of the near-infrared excess border (Meyer et al. 1997). On the other hand, such objects in the BRCs are plotted on either side of the near-infrared excess border, like CTTSs in the BRCs and also like CTTSs in Taurus.

In the Taurus molecular cloud, WTTSs are older than CTTSs. The ages of the TTSs in the BRCs were estimated on the $(I, I-J)$ color-magnitude diagram with the isochrone of [14]. I-magnitudes of the objects were taken from the USNO-B1.0 catalog. Because the extinction vector is relatively parallel to the isochrone on this diagram, it is possible to roughly estimate the age of the object. 
Table 2. $\mathrm{H} \alpha$ emission line stars.

\begin{tabular}{|c|c|c|c|c|c|c|c|c|c|c|c|}
\hline ID & \multicolumn{3}{|c|}{$\begin{array}{l}\text { RA [J2000] } \\
\text { h m s }\end{array}$} & \multicolumn{3}{|c|}{$\begin{array}{c}\mathrm{DEC}[\mathrm{J} 2000] \\
, \cdots\end{array}$} & \multirow{2}{*}{$\begin{array}{c}\mathrm{EW}(\mathrm{H} \alpha) \\
\AA\end{array}$} & \multirow[t]{2}{*}{$\begin{array}{c}J \\
\mathrm{mag}\end{array}$} & \multirow[t]{2}{*}{$\begin{array}{c}H \\
\text { mag }\end{array}$} & \multirow[t]{2}{*}{$\begin{array}{c}K \\
\text { mag }\end{array}$} & \multirow[t]{2}{*}{ Identification } \\
\hline & & & & & & & & & & & \\
\hline 1 & 5 & 23 & 32.21 & 33 & 27 & 43.6 & 16.8 & 14.36 & 13.29 & 12.41 & [PSM2011] 107 \\
\hline 2 & 5 & 23 & 15.81 & 33 & 27 & 43.5 & 3.8 & 15.17 & 14.25 & 13.96 & [PSM2011] 135, [CMP2012] 975 \\
\hline 3 & 5 & 22 & 54.07 & 33 & 26 & 33.0 & 1.2 & 14.35 & 13.55 & 13.25 & [PSM2011] 82, [CMP2012] 559 \\
\hline 4 & 5 & 22 & 51.19 & 33 & 26 & 32.9 & 11.4 & 15.96 & 15.19 & 14.59 & [MSB2007]37, [PSM2011] 851, [CMP2012] 465 \\
\hline 5 & 5 & 22 & 53.82 & 33 & 25 & 41.5 & 13.9 & 15.93 & 14.89 & 14.12 & [MSB2007]38, [PSM2011] 883, [CMP2012] 548 \\
\hline 6 & 5 & 22 & 52.30 & 33 & 24 & 7.4 & 1.7 & 13.58 & 12.71 & 12.25 & [PSM2011] 61, [CMP2012] 496 \\
\hline 7 & 5 & 22 & 51.90 & 33 & 23 & 59.3 & 1.1 & 14.61 & 14.21 & 13.65 & [PSM2011] 56 \\
\hline 8 & 5 & 22 & 51.04 & 33 & 25 & 47.1 & 2.5 & 13.08 & 12.34 & 11.75 & [MSB2007] 41, [PSM2011] 847, [CMP2012] 461 \\
\hline 9 & 5 & 22 & 54.15 & 33 & 24 & 58.0 & 2.7 & 15.15 & 14.46 & 14.20 & [PSM2011] 1168, [CMP2012] 563 \\
\hline 10 & 5 & 22 & 45.78 & 33 & 28 & 16.2 & 60 & 15.05 & 14.04 & 13.54 & [PSM2011] 177, [CMP2012] 294 \\
\hline 11 & 5 & 22 & 18.44 & 33 & 28 & 21.2 & 6.3 & 11.13 & 11.04 & 10.98 & NGC1893-256, [PSM2011] 1072, [CMP2012] 11 \\
\hline 12 & 5 & 22 & 51.04 & 33 & 25 & 47.1 & 1.1 & 13.08 & 12.34 & 11.75 & [MSB2007] 41, [PSM2011] 847, [CMP2012] 461 \\
\hline 13 & 5 & 22 & 43.02 & 33 & 25 & 5.4 & 24.5 & 11.65 & 11.31 & 10.92 & NGC1893-35, [MSB2007] 47, [PSM2011] 12 \\
\hline 14 & 5 & 22 & 43.78 & 33 & 25 & 25.8 & 40.1 & 12.38 & 10.77 & 9.42 & [MSB2007] 46, [PSM2011] 92, [CMP2012] 241 \\
\hline 15 & 5 & 22 & 38.78 & 33 & 22 & 5.4 & 8.6 & 14.68 & 13.70 & 13.08 & [PSM2011] 718, [CMP2012] 151 \\
\hline 16 & 5 & 22 & 46.84 & 33 & 29 & 27.9 & 9.6 & 14.83 & 13.9 & 13.36 & [MSB2007] 40, [PSM2011] 131, [CMP2012] 329 \\
\hline 17 & 5 & 22 & 49.35 & 33 & 29 & 2.1 & 7.1 & 14.99 & 14.06 & 13.57 & [PSM2011] 112, [CMP2012] 410 \\
\hline 18 & 5 & 22 & 49.57 & 33 & 30 & 1.5 & 28.8 & 14.42 & 13.45 & 12.95 & [PSM2011] 137, [CMP2012] 414 \\
\hline 19 & 5 & 23 & 9.33 & 33 & 30 & 2.3 & 35.7 & 10.79 & 10.41 & 10.01 & GGA 333, [PSM2011] 5 \\
\hline 20 & 5 & 23 & 6.32 & 33 & 31 & 1.7 & 6.4 & 14.74 & 13.82 & 13.24 & [PSM2011] 97, [CMP2012] 873 \\
\hline 21 & 5 & 23 & 4.96 & 33 & 31 & 50.1 & 1.5 & 15.14 & 14.38 & 13.89 & [PSM2011] 105, [CMP2012] 848 \\
\hline 22 & 5 & 22 & 52.23 & 33 & 29 & 58.0 & 6.8 & 12.68 & 11.91 & 11.35 & [MSB2007] 34, [PSM2011] 31, [CMP2012] 494 \\
\hline 23 & 5 & 23 & 3.32 & 33 & 29 & 25.1 & 1.7 & 15.46 & 14.41 & 13.72 & [MSB2007] 13, [PSM2011] 113, [CMP2012] 806 \\
\hline 24 & 5 & 23 & 2.76 & 33 & 29 & 40.2 & 8.8 & 15.47 & 14.60 & 13.90 & [MSB2007] 17, [PSM2011] 175, [CMP2012] 795 \\
\hline 25 & 5 & 23 & 0.07 & 33 & 30 & 39.0 & 21.7 & 14.34 & 13.42 & 12.97 & [PSM2011] 99, [CMP2012] 718 \\
\hline 26 & 5 & 22 & 58.11 & 33 & 30 & 41.0 & 9.3 & 13.84 & 13.30 & 12.63 & [MSB2007] 27, [PSM2011] 33 \\
\hline 27 & 5 & 23 & 13.59 & 33 & 29 & 44.0 & 5.2 & 15.34 & 14.48 & 13.93 & [PSM2011] 144, [CMP2012] 961 \\
\hline 28 & 5 & 23 & 1.07 & 33 & 29 & 25.1 & 30 & 15.97 & 14.89 & 14.19 & [MSB2007] 21, [PSM2011] 253, [CMP2012] 743 \\
\hline 29 & 5 & 23 & 9.95 & 33 & 29 & 8.8 & 12.6 & 15.40 & 14.11 & 13.20 & [MSB2007] 2, [PSM2011] 140, [CMP2012] 924 \\
\hline 19 & 5 & 23 & 9.33 & 33 & 30 & 2.3 & 35.7 & 10.79 & 10.41 & 10.01 & GGA 333, [PSM2011] 5 \\
\hline 20 & 5 & 23 & 6.32 & 33 & 31 & 1.7 & 6.4 & 14.74 & 13.82 & 13.24 & [PSM2011] 97, [CMP2012] 873 \\
\hline 21 & 5 & 23 & 4.96 & 33 & 31 & 50.1 & 1.5 & 15.14 & 14.38 & 13.89 & [PSM2011] 105, [CMP2012] 848 \\
\hline 22 & 5 & 22 & 52.23 & 33 & 29 & 58.0 & 6.8 & 12.68 & 11.91 & 11.35 & [MSB2007] 34, [PSM2011] 31, [CMP2012] 494 \\
\hline 23 & 5 & 23 & 3.32 & 33 & 29 & 25.1 & 1.7 & 15.46 & 14.41 & 13.72 & [MSB2007] 13, [PSM2011] 113, [CMP2012] 806 \\
\hline
\end{tabular}




\section{Continued}

\begin{tabular}{|c|c|c|c|c|c|c|c|c|c|c|c|}
\hline \multirow{2}{*}{$\begin{array}{l}\text { ID } \\
24\end{array}$} & \multicolumn{3}{|c|}{$\begin{array}{l}\text { RA [J2000] } \\
\text { h m s }\end{array}$} & \multicolumn{3}{|c|}{ DEC [J2000] } & \multirow{2}{*}{$\begin{array}{c}\mathrm{EW}(\mathrm{H} \alpha) \\
\AA \\
8.8\end{array}$} & \multirow{2}{*}{$\begin{array}{c}\begin{array}{c}\text { J mag } \\
\text { mag }\end{array} \\
15.47\end{array}$} & \multirow{2}{*}{$\begin{array}{c}\begin{array}{c}\text { H mag } \\
\text { mag }\end{array} \\
14.60\end{array}$} & \multirow{2}{*}{$\begin{array}{c}\begin{array}{c}\text { K mag } \\
\text { mag }\end{array} \\
13.90\end{array}$} & Identification \\
\hline & 5 & 23 & 2.76 & 33 & 29 & 40.2 & & & & & [MSB2007] 17, [PSM2011] 175, [CMP2012] 795 \\
\hline 25 & 5 & 23 & 0.07 & 33 & 30 & 39.0 & 21.7 & 14.34 & 13.42 & 12.97 & [PSM2011] 99, [CMP2012] 718 \\
\hline 26 & 5 & 22 & 58.11 & 33 & 30 & 41.0 & 9.3 & 13.84 & 13.30 & 12.63 & [MSB2007] 27, [PSM2011] 33 \\
\hline 27 & 5 & 23 & 13.59 & 33 & 29 & 44.0 & 5.2 & 15.34 & 14.48 & 13.93 & [PSM2011] 144, [CMP2012] 961 \\
\hline 28 & 5 & 23 & 1.07 & 33 & 29 & 25.1 & 30 & 15.97 & 14.89 & 14.19 & [MSB2007] 21, [PSM2011] 253, [CMP2012] 743 \\
\hline 29 & 5 & 23 & 9.95 & 33 & 29 & 8.8 & 12.6 & 15.40 & 14.11 & 13.20 & [MSB2007] 2, [PSM2011] 140, [CMP2012] 924 \\
\hline 30 & 5 & 23 & 3.82 & 33 & 29 & 24.2 & 2 & 14.80 & 13.90 & 13.30 & [MSB2007] 12, [PSM2011] 114, [CMP2012] 819 \\
\hline 31 & 5 & 23 & 4.05 & 33 & 29 & 48.4 & 26.3 & 15.03 & 13.95 & 13.15 & [MSB2007] 16, [PSM2011] 201, [CMP2012] 825 \\
\hline \multicolumn{11}{|c|}{ BRC 17} & \\
\hline 1 & 5 & 31 & 23.12 & 12 & 10 & 33.7 & 6.9 & 13.17 & 12.46 & 12.02 & \\
\hline 2 & 5 & 31 & 26.94 & 12 & 10 & 20.5 & 2.7 & 11.96 & 11.25 & 10.89 & \\
\hline 3 & 5 & 31 & 23.59 & 12 & 9 & 43.9 & 5.2 & 10.42 & 9.62 & 9.19 & HI Ori, HBC 93, [DM99] 143 \\
\hline 4 & 5 & 31 & 28.05 & 12 & 9 & 10.3 & 16.8 & 9.41 & 8.31 & 7.34 & HK Ori, HBC 94 \\
\hline 5 & 5 & 31 & 15.51 & 12 & 11 & 23.7 & 2.5 & 12.10 & 11.21 & 10.72 & [DM99] 136 \\
\hline 6 & 5 & 31 & 19.46 & 12 & 9 & 15.3 & 2.2 & 11.99 & 10.78 & 10.09 & \\
\hline 7 & 5 & 30 & 51.70 & 12 & 8 & 36.7 & 26.5 & 11.64 & 10.87 & 10.45 & V4480 Ori, HBC 91, [DM99]125 \\
\hline 8 & 5 & 31 & 12.49 & 12 & 7 & 54.5 & 2 & 13.29 & 12.52 & 12.21 & \\
\hline 9 & 5 & 31 & 21.91 & 11 & 54 & 56.7 & 2.2 & 13.60 & 12.96 & 12.69 & \\
\hline \multicolumn{11}{|c|}{ BRC 18} & \\
\hline 1 & 5 & 44 & 19.40 & 9 & 16 & 19.3 & 3.5 & 13.12 & 12.39 & 12.03 & \\
\hline 2 & 5 & 44 & 22.89 & 9 & 10 & 35.3 & 2.5 & 12.45 & 11.72 & 11.45 & [DM99] 247 \\
\hline 3 & 5 & 44 & 23.21 & 9 & 12 & 3.9 & 1.4 & 10.00 & 9.36 & 8.95 & [DM99] 248 \\
\hline 4 & 5 & 44 & 37.32 & 9 & 11 & 59.1 & 3.8 & 12.95 & 12.33 & 12.05 & \\
\hline 5 & 5 & 44 & 37.02 & 9 & 13 & 20.0 & 15.4 & 12.33 & 11.66 & 11.33 & [DM99] 252 \\
\hline 6 & 5 & 44 & 30.23 & 9 & 12 & 23.8 & 8.1 & 14.13 & 13.36 & 12.91 & \\
\hline 7 & 5 & 44 & 17.35 & 9 & 10 & 59.2 & 17 & 10.79 & 10.04 & 9.62 & V630 Ori, [DM99] 244 \\
\hline 8 & 5 & 44 & 25.73 & 9 & 12 & 1.3 & 1.9 & 13.07 & 12.35 & 12.09 & \\
\hline 9 & 5 & 44 & 10.04 & 9 & 8 & 40.8 & 43.2 & 13.85 & 13.12 & 12.64 & \\
\hline 10 & 5 & 44 & 14.86 & 9 & 8 & 8.2 & 9.4 & 12.67 & 11.97 & 11.67 & [DM99] 243 \\
\hline 11 & 5 & 44 & 7.26 & 9 & 6 & 38.1 & 41.9 & 11.66 & 10.94 & 10.68 & V629 Ori, [DM99] 240 \\
\hline 12 & 5 & 44 & 28.66 & 9 & 6 & 18.6 & 8.8 & 14.43 & 13.81 & 13.46 & \\
\hline 13 & 5 & 44 & 8.99 & 9 & 9 & 14.8 & 3.4 & 11.10 & 10.03 & 9.25 & QR Ori, [DM99] 241 \\
\hline 14 & 5 & 44 & 12.77 & 9 & 5 & 10.5 & 10.5 & 13.26 & 12.59 & 12.24 & \\
\hline 15 & 5 & 43 & 56.59 & 9 & 18 & 16.9 & 26.8 & 12.85 & 12.04 & 11.67 & [DM99] 238 \\
\hline 16 & 5 & 43 & 31.22 & 9 & 17 & 46.3 & 10.8 & 12.88 & 12.30 & 11.97 & \\
\hline
\end{tabular}




\section{Continued}

\begin{tabular}{|c|c|c|c|c|c|c|c|c|c|c|c|}
\hline \multirow{2}{*}{$\begin{array}{l}\text { ID } \\
17\end{array}$} & \multicolumn{3}{|c|}{$\begin{array}{l}\text { RA [J2000] } \\
\text { h m s }\end{array}$} & \multicolumn{3}{|c|}{$\begin{array}{c}\text { DEC [J2000] } \\
, ",\end{array}$} & \multirow{2}{*}{$\begin{array}{c}\mathrm{EW}(\mathrm{H} \alpha) \\
\AA \\
38.8\end{array}$} & \multirow{2}{*}{$\begin{array}{c}\begin{array}{c}\text { J mag } \\
\text { mag }\end{array} \\
12.34\end{array}$} & \multirow{2}{*}{$\begin{array}{c}\begin{array}{c}\text { H mag } \\
\text { mag }\end{array} \\
11.57\end{array}$} & \multirow{2}{*}{$\begin{array}{c}\begin{array}{c}\mathrm{K} \text { mag } \\
\mathrm{mag}\end{array} \\
11.17\end{array}$} & \multirow[t]{2}{*}{ Identification } \\
\hline & 5 & 43 & 57.00 & 9 & 16 & 24.0 & & & & & \\
\hline 18 & 5 & 43 & 50.67 & 9 & 12 & 25.0 & 10.3 & 13.33 & 12.37 & 11.82 & \\
\hline 19 & 5 & 43 & 39.59 & 9 & 10 & 40.8 & 4.9 & 13.34 & 12.66 & 12.35 & \\
\hline 20 & 5 & 43 & 51.36 & 9 & 13 & 40.6 & 66.9 & 14.62 & 13.98 & 13.74 & \\
\hline 21 & 5 & 43 & 32.20 & 9 & 9 & 24.0 & 7.2 & 11.29 & 10.55 & 10.19 & V626 Ori, [DM99]231 \\
\hline 22 & 5 & 43 & 44.50 & 9 & 8 & 1.2 & 20.8 & 14.57 & 12.62 & 11.09 & \\
\hline 23 & 5 & 43 & 53.50 & 9 & 7 & 11.8 & 42 & 13.09 & 12.23 & 11.71 & \\
\hline 24 & 5 & 43 & 20.92 & 9 & 6 & 7.1 & 5 & 10.07 & 9.13 & 8.41 & V625 Ori, [DM99]227 \\
\hline \multirow[t]{2}{*}{25} & 5 & 43 & 52.20 & 9 & 6 & 51.4 & 1.8 & 14.45 & 13.82 & 13.56 & \\
\hline & \multicolumn{10}{|c|}{ BRC 19} & \\
\hline 1 & 5 & 34 & 34.24 & -2 & 58 & 16.6 & 55 & 12.77 & 11.67 & 11.03 & Haro 5-83 \\
\hline \multirow[t]{2}{*}{2} & 5 & 34 & 20.38 & -2 & 57 & 46.9 & 3.1 & 13.75 & 12.27 & 11.27 & V1945 Ori \\
\hline & \multicolumn{10}{|c|}{ BRC 24} & \\
\hline 1 & 6 & 34 & 25.74 & 4 & 28 & 15.8 & 20.6 & 14.57 & 13.55 & 12.76 & [WFT2009] RMCX 324 \\
\hline 2 & 6 & 34 & 41.04 & 4 & 27 & 9.9 & 15.9 & 14.82 & 13.50 & 12.68 & \\
\hline 3 & 6 & 33 & 50.40 & 4 & 32 & 55.9 & 3.5 & 13.11 & 12.42 & 11.98 & \\
\hline 4 & 6 & 34 & 2.21 & 4 & 30 & 7.6 & 9.9 & 14.43 & 13.49 & 13.00 & [WFT2009] RMCX 189 \\
\hline 5 & 6 & 34 & 17.07 & 4 & 27 & 35.3 & 22.5 & 14.72 & 13.53 & 12.69 & \\
\hline 6 & 6 & 34 & 4.22 & 4 & 34 & 34.1 & 9.9 & 14.45 & 13.44 & 12.86 & [WFT2009] RMCX 199 \\
\hline 7 & 6 & 34 & 3.41 & 4 & 34 & 8.8 & 22 & 13.24 & 12.36 & 11.72 & [WFT2009] RMCX 195 \\
\hline 8 & 6 & 33 & 58.01 & 4 & 33 & 31.3 & 20.2 & 14.75 & 13.71 & 12.97 & [WFT2009] RMCX 179 \\
\hline 9 & 6 & 33 & 55.73 & 4 & 28 & 23.4 & 14.4 & 15.48 & 14.41 & 13.85 & \\
\hline 10 & 6 & 33 & 54.60 & 4 & 29 & 41.6 & 6 & 15.35 & 13.87 & 12.88 & \\
\hline 11 & 6 & 34 & 8.90 & 4 & 29 & 38.5 & 13.7 & 15.15 & 14.13 & 13.64 & [WFT2009] RMCX 233 \\
\hline 12 & 6 & 33 & 45.75 & 4 & 31 & 3.5 & 25.4 & 15.24 & 14.08 & 13.56 & \\
\hline 13 & 6 & 33 & 56.09 & 4 & 31 & 19.3 & 32.1 & 14.65 & 13.65 & 13.11 & [WFT2009] RMCX 172 \\
\hline 14 & 6 & 34 & 2.85 & 4 & 34 & 51.0 & 2.4 & 13.55 & 12.68 & 12.20 & [WFT2009] RMCX 190 \\
\hline 15 & 6 & 33 & 41.16 & 4 & 37 & 5.1 & 3.8 & 14.01 & 12.94 & 12.17 & [WFT2009] RMCX 149 \\
\hline 16 & 6 & 32 & 35.75 & 4 & 46 & 30.4 & 23 & 14.01 & 13.10 & 12.56 & [BNM2013] 66.0115 \\
\hline 4 & 6 & 34 & 2.21 & 4 & 30 & 7.6 & 9.9 & 14.43 & 13.49 & 13.00 & [WFT2009] RMCX 189 \\
\hline 5 & 6 & 34 & 17.07 & 4 & 27 & 35.3 & 22.5 & 14.72 & 13.53 & 12.69 & \\
\hline 6 & 6 & 34 & 4.22 & 4 & 34 & 34.1 & 9.9 & 14.45 & 13.44 & 12.86 & [WFT2009] RMCX 199 \\
\hline 7 & 6 & 34 & 3.41 & 4 & 34 & 8.8 & 22 & 13.24 & 12.36 & 11.72 & [WFT2009] RMCX 195 \\
\hline 8 & 6 & 33 & 58.01 & 4 & 33 & 31.3 & 20.2 & 14.75 & 13.71 & 12.97 & [WFT2009] RMCX 179 \\
\hline 9 & 6 & 33 & 55.73 & 4 & 28 & 23.4 & 14.4 & 15.48 & 14.41 & 13.85 & \\
\hline
\end{tabular}




\section{Continued}

\begin{tabular}{|c|c|c|c|c|c|c|c|c|c|c|c|}
\hline \multirow{2}{*}{$\frac{\text { ID }}{10}$} & \multicolumn{3}{|c|}{$\begin{array}{l}\text { RA [J2000] } \\
\text { h m s }\end{array}$} & \multicolumn{3}{|c|}{$\begin{array}{c}\mathrm{DEC}[\mathrm{J} 2000] \\
, \cdots\end{array}$} & \multirow{2}{*}{$\begin{array}{c}\text { EW(Ha) } \\
\AA \\
6\end{array}$} & \multirow{2}{*}{$\begin{array}{c}\begin{array}{c}\text { J mag } \\
\text { mag }\end{array} \\
15.35\end{array}$} & \multirow{2}{*}{$\begin{array}{c}\begin{array}{c}\text { H mag } \\
\text { mag }\end{array} \\
13.87\end{array}$} & \multirow{2}{*}{$\begin{array}{c}\begin{array}{c}\text { K mag } \\
\text { mag }\end{array} \\
12.88\end{array}$} & \multirow[t]{2}{*}{ Identification } \\
\hline & 6 & 33 & 54.60 & 4 & 29 & 41.6 & & & & & \\
\hline 11 & 6 & 34 & 8.90 & 4 & 29 & 38.5 & 13.7 & 15.15 & 14.13 & 13.64 & [WFT2009] RMCX 233 \\
\hline 12 & 6 & 33 & 45.75 & 4 & 31 & 3.5 & 25.4 & 15.24 & 14.08 & 13.56 & \\
\hline 13 & 6 & 33 & 56.09 & 4 & 31 & 19.3 & 32.1 & 14.65 & 13.65 & 13.11 & [WFT2009] RMCX 172 \\
\hline 14 & 6 & 34 & 2.85 & 4 & 34 & 51.0 & 2.4 & 13.55 & 12.68 & 12.20 & [WFT2009] RMCX 190 \\
\hline 15 & 6 & 33 & 41.16 & 4 & 37 & 5.1 & 3.8 & 14.01 & 12.94 & 12.17 & [WFT2009] RMCX 149 \\
\hline 16 & 6 & 32 & 35.75 & 4 & 46 & 30.4 & 23 & 14.01 & 13.10 & 12.56 & [BNM2013] 66.0115 \\
\hline 17 & 6 & 32 & 34.95 & 4 & 44 & 39.2 & 32.8 & 10.11 & 9.90 & 9.53 & NGC2244 279 \\
\hline 18 & 6 & 32 & 45.11 & 4 & 45 & 23.1 & 15.5 & 13.33 & 12.29 & 11.57 & [WTF2008] Main 878 \\
\hline 19 & 6 & 32 & 31.01 & 4 & 50 & 6.0 & 1.5 & 12.07 & 11.57 & 11.09 & NGC2244 269 \\
\hline 20 & 6 & 32 & 31.44 & 4 & 42 & 34.0 & 0.5 & 11.74 & 11.03 & 10.83 & [WTF2008] Main 831 \\
\hline 21 & 6 & 32 & 50.74 & 4 & 44 & 47.6 & 1.3 & 12.94 & 12.09 & 11.70 & [WTF2008] Main 898 \\
\hline 22 & 6 & 32 & 1.83 & 4 & 53 & 38.6 & 6.5 & 14.26 & 13.32 & 12.91 & [WTF2008] Main 498 \\
\hline 23 & 6 & 32 & 7.82 & 4 & 52 & 28.4 & 18.3 & 14.03 & 13.21 & 12.76 & [WTF2008] Main 590 \\
\hline 24 & 6 & 32 & 4.66 & 4 & 54 & 51.5 & 2.8 & 13.80 & 12.92 & 12.54 & [WTF2008] Main 541 \\
\hline 25 & 6 & 32 & 4.53 & 4 & 53 & 25.0 & 2.7 & 13.59 & 12.71 & 12.23 & [WTF2008] Main 536 \\
\hline 26 & 6 & 31 & 41.02 & 4 & 54 & 47.9 & 8.3 & 14.18 & 13.36 & 12.95 & [WTF2008] Main 133 \\
\hline 27 & 6 & 31 & 43.85 & 5 & 2 & 57.5 & 4 & 13.27 & 12.38 & 11.97 & [WTF2008] Main 169 \\
\hline 28 & 6 & 31 & 49.26 & 4 & 57 & 0.9 & 5.4 & 13.7 & 12.85 & 12.27 & [WTF2008] Main 254 \\
\hline 29 & 6 & 31 & 51.65 & 4 & 55 & 5.2 & 6.7 & 13.91 & 13.01 & 12.45 & V547 Mon \\
\hline 30 & 6 & 31 & 29.76 & 4 & 54 & 49.1 & 8.8 & 11.45 & 10.89 & 9.69 & GGA 395, NGC2244 74 \\
\hline 31 & 6 & 31 & 39.62 & 4 & 59 & 45.1 & 23.5 & 14.12 & 13.13 & 12.65 & [WTF2008] Main 124 \\
\hline 32 & 6 & 31 & 39.88 & 4 & 56 & 39.1 & 17.8 & 15.05 & 14.10 & 13.62 & \\
\hline 33 & 6 & 31 & 20.87 & 5 & 4 & 8.5 & 3.8 & 14.07 & 13.23 & 12.71 & [BNM2013] 66.03296 \\
\hline 34 & 6 & 31 & 48.31 & 4 & 58 & 20.3 & 5.8 & 12.43 & 11.57 & 11.08 & [WTF2008] Main 243 \\
\hline 35 & 6 & 31 & 29.52 & 4 & 54 & 34.2 & 8.1 & 14.92 & 14.11 & 13.72 & [WTF2008] Main 43 \\
\hline 36 & 6 & 30 & 42.71 & 4 & 55 & 31.4 & 1.7 & 11.23 & 10.47 & 9.75 & V539 Mon \\
\hline 37 & 6 & 31 & 0.55 & 4 & 58 & 7.3 & 8.4 & 14.64 & 13.76 & 13.18 & [WFT2010] 126 \\
\hline 38 & 6 & 31 & 30.96 & 5 & 6 & 58.5 & 28.6 & 12.88 & 12.08 & 11.60 & \\
\hline 39 & 6 & 31 & 40.00 & 5 & 5 & 56.4 & 6.3 & 9.68 & 8.96 & 8.27 & NGC2244 106 \\
\hline 40 & 6 & 31 & 2.88 & 5 & 3 & 49.3 & 8.9 & 13.74 & 12.84 & 12.38 & [WFT2010] 134 \\
\hline 41 & 6 & 31 & 12.63 & 5 & 5 & 2.6 & 3.6 & 14.42 & 13.30 & 12.62 & \\
\hline \multicolumn{11}{|c|}{ BRC 25} & \\
\hline 1 & 6 & 41 & 1.53 & 10 & 14 & 56.1 & 20.7 & 12.93 & 12.18 & 11.66 & $\mathrm{LkH} \alpha 46$ \\
\hline 2 & 6 & 41 & 7.48 & 10 & 15 & 4.5 & 3.3 & 13.24 & 12.47 & 12.15 & ESO-H $\alpha 493$ \\
\hline
\end{tabular}




\section{Continued}

\begin{tabular}{|c|c|c|c|c|c|c|c|c|c|c|c|}
\hline 3 & 6 & 41 & 9.85 & 10 & 15 & 2.6 & 11.1 & 13.19 & 11.80 & 10.85 & ESO-H $\alpha 504$ \\
\hline 4 & 6 & 41 & 7.01 & 10 & 16 & 28.9 & 1.3 & 12.99 & 12.32 & 12.06 & \\
\hline 5 & 6 & 40 & 59.79 & 10 & 2 & 12.6 & 15.5 & 14.66 & 13.82 & 13.50 & ESO-H $\alpha 454$ \\
\hline 6 & 6 & 41 & 2.86 & 10 & 7 & 11.9 & 4.5 & 13.98 & 13.22 & 12.87 & ESO-H $\alpha 468$ \\
\hline 7 & 6 & 41 & 7.26 & 9 & 58 & 31.2 & 0.3 & 12.54 & 11.87 & 11.68 & V609 Mon \\
\hline 8 & 6 & 40 & 49.21 & 9 & 57 & 38.8 & 12.4 & 13.57 & 12.74 & 12.37 & ESO-H $\alpha 415$ \\
\hline 9 & 6 & 41 & 17.25 & 9 & 54 & 32.4 & 25.6 & 12.83 & 12.05 & 11.55 & IQ Mon \\
\hline 10 & 6 & 40 & 49.75 & 9 & 52 & 58.6 & 2.7 & 14.20 & 13.56 & 13.33 & V343 Mon \\
\hline 11 & 6 & 40 & 50.59 & 9 & 54 & 57.3 & 6.1 & 12.47 & 11.69 & 11.28 & $\mathrm{LkH} \alpha 31$ \\
\hline 12 & 6 & 40 & 52.55 & 9 & 52 & 6.0 & 2 & 12.69 & 11.97 & 11.72 & ESO-H $\alpha 425$ \\
\hline 13 & 6 & 41 & 12.58 & 9 & 52 & 31.2 & 9.6 & 11.49 & 10.69 & 10.21 & MM Mon \\
\hline 14 & 6 & 40 & 59.45 & 9 & 59 & 45.5 & 4.9 & 12.78 & 12.09 & 11.69 & V602 Mon \\
\hline 15 & 6 & 40 & 54.88 & 9 & 53 & 12.4 & 5.5 & 13.92 & 13.09 & 12.40 & ESO-H $\alpha 435$ \\
\hline 16 & 6 & 40 & 46.95 & 9 & 52 & 40.6 & 42 & 14.75 & 14.06 & 13.41 & ESO-H $\alpha 405$ \\
\hline 17 & 6 & 40 & 54.11 & 95 & 52 & 24.6 & 28.9 & 15.34 & 14.88 & 14.33 & ESO-H $\alpha 432$ \\
\hline 18 & 6 & 40 & 54.20 & 9 & 55 & 52.0 & 32 & 13.70 & 12.94 & 12.76 & NGC2264 SBL 219 \\
\hline \multicolumn{11}{|c|}{ BRC 26} & \\
\hline 1 & 7 & 3 & 40.75 & -11 & 46 & 16.6 & 22.7 & 15.24 & 14.06 & 13.21 & \\
\hline 2 & 7 & 3 & 48.22 & -11 & 41 & 46.0 & 5.2 & 13.61 & 12.84 & 12.55 & \\
\hline 3 & 7 & 4 & 7.18 & -11 & 40 & 32.7 & 3.9 & 14.08 & 13.87 & 13.73 & \\
\hline 4 & 7 & 3 & 49.91 & -11 & 31 & 17.0 & 8.2 & 14.03 & 13.20 & 12.95 & \\
\hline 5 & 7 & 3 & 54.00 & -11 & 32 & 47.9 & 5.2 & 12.34 & 11.61 & 11.18 & \\
\hline 6 & 7 & 3 & 54.03 & -11 & 32 & 37.2 & 63.1 & 14.57 & 13.7 & 13.29 & \\
\hline 7 & 7 & 3 & 58.35 & -11 & 33 & 34.6 & 19.1 & 14.92 & 13.93 & 13.52 & \\
\hline 8 & 7 & 4 & 1.33 & -11 & 36 & 17.5 & 26.3 & 14.77 & 13.93 & 13.42 & \\
\hline 9 & 7 & 4 & 0.68 & -11 & 37 & 49.3 & 12.8 & 13.98 & 13.23 & 12.93 & \\
\hline 10 & 7 & 3 & 38.52 & -11 & 31 & 51.9 & 22.8 & 14.22 & 13.28 & 12.75 & \\
\hline 11 & 7 & 3 & 43.68 & -11 & 36 & 56.6 & 33.3 & 14.89 & 13.92 & 13.66 & \\
\hline 12 & 7 & 4 & 4.70 & -11 & 31 & 15.9 & 24.5 & 15.57 & 14.66 & 14.38 & \\
\hline 13 & 7 & 3 & 58.06 & -11 & 32 & 39.9 & 3.4 & 14.10 & 13.23 & 12.93 & \\
\hline 14 & 7 & 3 & 50.34 & -11 & 32 & 51.4 & 16.3 & 15.81 & 14.92 & 14.30 & [CPD2009] J070350.34-113251.4 \\
\hline 15 & 7 & 4 & 0.41 & -11 & 33 & 59.6 & 2.4 & 12.10 & 10.92 & 10.19 & [CPD2009] J070400.41-113359.5 \\
\hline 16 & 7 & 3 & 56.71 & -11 & 35 & 9.6 & 23.3 & 14.34 & 12.95 & 12.11 & [CPD2009] J070356.71-113509.6 \\
\hline 17 & 7 & 4 & 3.94 & -11 & 35 & 57.1 & 11.6 & 14.51 & 13.63 & 13.21 & \\
\hline 18 & 7 & 4 & 5.53 & -11 & 39 & 52.4 & 28.2 & 15.62 & 14.72 & 14.20 & \\
\hline 19 & 7 & 3 & 48.98 & -11 & 37 & 19.4 & 20 & 15.42 & 14.58 & 14.24 & \\
\hline
\end{tabular}




\section{Continued}

\begin{tabular}{|c|c|c|c|c|c|c|c|c|c|c|c|}
\hline \multirow[t]{2}{*}{ ID } & \multicolumn{3}{|c|}{$\begin{array}{l}\text { RA [J2000] } \\
\text { h m s }\end{array}$} & \multicolumn{3}{|c|}{$\begin{array}{c}\text { DEC }[J 2000] \\
\circ, "\end{array}$} & \multirow{2}{*}{$\begin{array}{c}\mathrm{EW}(\mathrm{H} \alpha) \\
\AA \\
\text { RC } 27\end{array}$} & \multirow[t]{2}{*}{$\begin{array}{c}J \mathrm{mag} \\
\mathrm{mag}\end{array}$} & \multirow[t]{2}{*}{$\begin{array}{c}H \text { mag } \\
\text { mag }\end{array}$} & \multirow[t]{2}{*}{$\begin{array}{c}K \mathrm{mag} \\
\mathrm{mag}\end{array}$} & \multirow[t]{2}{*}{ Identification } \\
\hline & & & & & & & & & & & \\
\hline 1 & 7 & 4 & 8.04 & -11 & 23 & 54.7 & 16.9 & 13.12 & 12.44 & 12.20 & [OSP2002] BRC 2722 \\
\hline 2 & 7 & 4 & 6.70 & -11 & 26 & 8.5 & 17 & 10.76 & 10.02 & 9.12 & $\mathrm{LkH} \alpha 220$ \\
\hline 3 & 7 & 4 & 9.96 & -11 & 23 & 16.4 & 18 & 11.79 & 10.71 & 9.83 & [OSP2002] BRC 2725 \\
\hline 4 & 7 & 3 & 53.04 & -11 & 29 & 35.3 & 10.9 & 12.36 & 11.54 & 10.95 & $\mathrm{LkH} \alpha 219$ \\
\hline 5 & 7 & 3 & 54.03 & -11 & 32 & 37.2 & 88.3 & 14.57 & 13.7 & 13.29 & \\
\hline 6 & 7 & 3 & 49.9 & -11 & 31 & 17.0 & 10.1 & 14.03 & 13.20 & 12.95 & \\
\hline 7 & 7 & 3 & 45.76 & -11 & 23 & 14.9 & 5.8 & 13.59 & 12.59 & 12.19 & \\
\hline 8 & 7 & 4 & 4.27 & -11 & 23 & 55.7 & 100 & 15.01 & 14.02 & 13.54 & [OSP2002] BRC 2712 \\
\hline 9 & 7 & 4 & 5.93 & -11 & 23 & 58.7 & 6.1 & 14.42 & 13.47 & 12.93 & [OSP2002] BRC 2716 \\
\hline 10 & 7 & 4 & 12.93 & -11 & 24 & 3.2 & 5.9 & 15.37 & 14.39 & 13.91 & [OSP2002] BRC 2728 \\
\hline 11 & 7 & 3 & 47.58 & -11 & 22 & 33.9 & 3.5 & 13.93 & 13.15 & 12.85 & \\
\hline 12 & 7 & 3 & 57.05 & -11 & 30 & 16.9 & 131.8 & 15.30 & 14.63 & 14.30 & \\
\hline 13 & 7 & 4 & 22.75 & -11 & 27 & 50.9 & 12.8 & 14.24 & 13.36 & 12.95 & \\
\hline 14 & 7 & 4 & 20.87 & -11 & 29 & 36.2 & 18.6 & 13.80 & 12.88 & 12.29 & [CPD2009] J070420.87-112936.2 \\
\hline 15 & 7 & 4 & 17.79 & -11 & 28 & 21.2 & 23 & 14.50 & 13.75 & 13.3 & \\
\hline 16 & 7 & 4 & 45.00 & -11 & 27 & 58.9 & 28 & 14.86 & 14.16 & 13.80 & \\
\hline 17 & 7 & 4 & 23.25 & -11 & 24 & 17.3 & 5.8 & 13.45 & 12.64 & 12.19 & \\
\hline 18 & 7 & 4 & 16.80 & -11 & 24 & 32.4 & 46.2 & 14.12 & 13.21 & 12.60 & [CPD2009] 112 \\
\hline 19 & 7 & 4 & 40.79 & -11 & 25 & 37.0 & 7 & 14.38 & 13.57 & 13.26 & \\
\hline 20 & 7 & 4 & 13.53 & -11 & 24 & 55.8 & 15.3 & 12.19 & 11.29 & 10.78 & [OSP2002] BRC 2729 \\
\hline 21 & 7 & 4 & 26.26 & -11 & 31 & 20.7 & 17.8 & 12.47 & 11.60 & 11.15 & $\mathrm{LkH} \alpha 222$ \\
\hline \multicolumn{11}{|c|}{ BRC 45} & \\
\hline 1 & 7 & 18 & 36.26 & -22 & 7 & 14.6 & 9.7 & 15.71 & 14.87 & 14.67 & \\
\hline 2 & 7 & 18 & 22.93 & -22 & 4 & 14.5 & 10.5 & 13.3 & 12.45 & 11.84 & \\
\hline 3 & 7 & 18 & 28.46 & -22 & 5 & 15 & 31.5 & 15.07 & 14.03 & 13.36 & \\
\hline 4 & 7 & 18 & 39.85 & -22 & 0 & 42.2 & 10.3 & 15.50 & 14.73 & 14.51 & \\
\hline 5 & 7 & 19 & 2.80 & -22 & 1 & 4.6 & 38.6 & 13.80 & 12.97 & 12.24 & \\
\hline 6 & 7 & 18 & 56.73 & -22 & 1 & 5.3 & 12.8 & 14.89 & 14.09 & 13.55 & \\
\hline 7 & 7 & 18 & 57.67 & -22 & 1 & 36.4 & 31.1 & 14.66 & 13.47 & 12.83 & \\
\hline
\end{tabular}

[CDP2009]: [17]; [CMP2012]: [18]; [DM99]: [19]; [BNM2013]: [20]; [MSB2007]: [21]; [OSP2002]: [10]; [PSM2011]: [22]; [WFT2009]: [23]; [WTF2008]: [24].

It is indicated that majority of the objects have the age between $1 \mathrm{Myr}$ and 10 Myr. We did not find any differences in the ages between the objects with a strong $\mathrm{H} \alpha$ emission line and the objects with a weak $\mathrm{H} \alpha$ emission line.

Masses of the TTSs were estimated with the isochrones of [14] [15] and [16] 


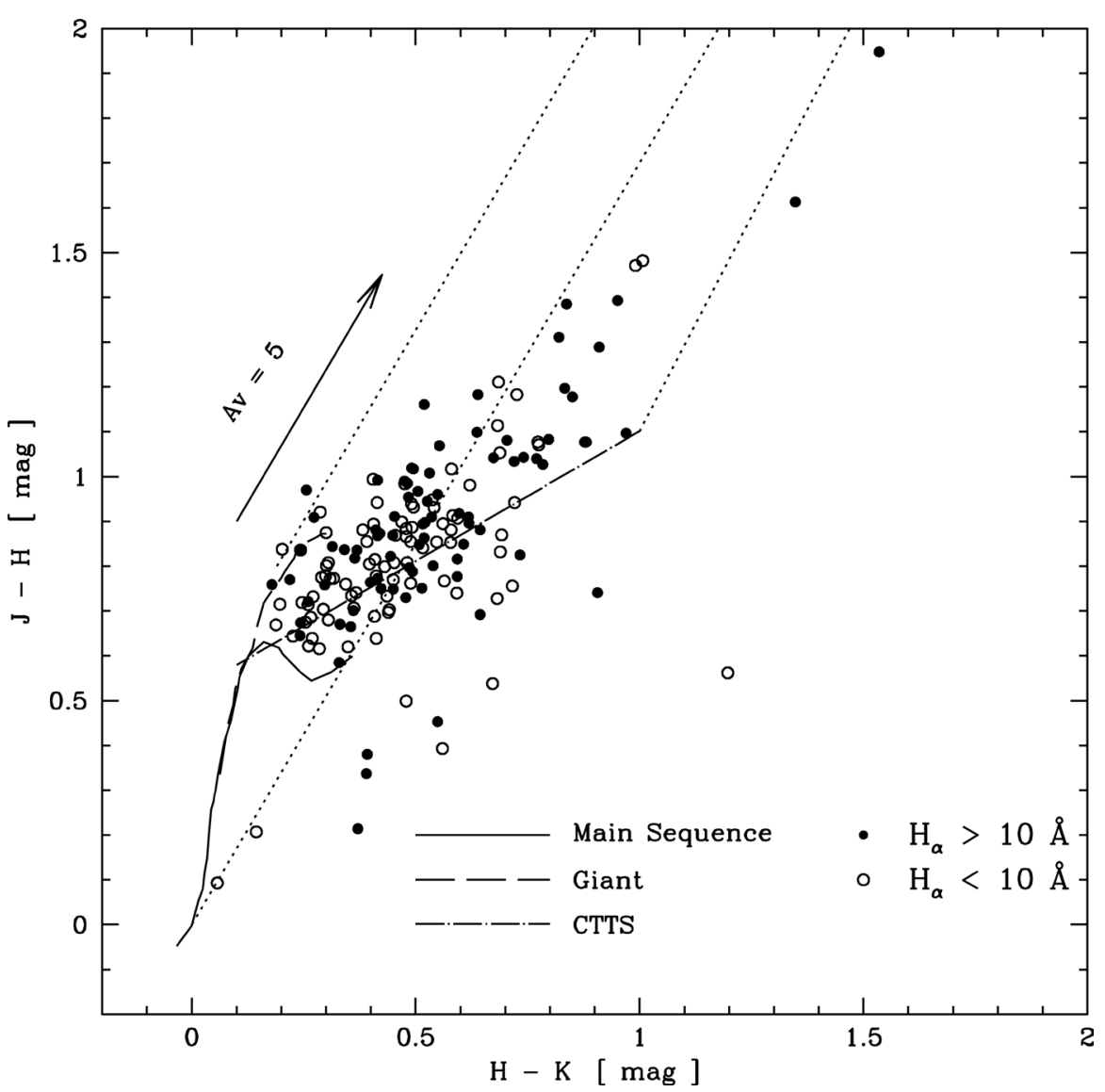

Figure 1. Near-infrared color-color diagram of the $\mathrm{H} \alpha$ emission line stars in the BRCs. A certain portion of stars with a weak $\mathrm{H} \alpha$ emission line show intrinsic near-infrared excess.

on the $(J, J-H)$ color-magnitude diagram. It is revealed that most of the objects have mass between $0.5 \mathrm{M}_{\odot}$ and $2 \mathrm{M}_{\odot}$.

\section{Discussion}

We identified $\mathrm{H} \alpha$ emission line stars in the BRCs. Some objects show intrinsic near-infrared excess and the others do not. We investigated the relationship between the intrinsic near-infrared excess and the $\mathrm{H} \alpha$ equivalent widths (Figure 2). The intersection of the reddening vector originating from the observed $J H K$ colors of the TTSs and the dereddened CTTS line represents the amount of the intrinsic near-infrared excess of the TTS. Zero point of the intrinsic near-infrared excess is defined as the intersection of the near-infrared excess border and the dereddened CTTS line. We also defined the point of unity of the intrinsic near-infrared excess as the reddest intrinsic color of CTTSs (Meyer et al. 1997). The object with the intrinsic near-infrared excess $>0$ has an intrinsic near-infrared excess. CTTSs and WTTSs are classified by the $\mathrm{H} \alpha$ equivalent width. We classified the objects into four types. Type 1 object has the $\mathrm{H} \alpha$ emission line with its equivalent width less than $10 \AA$ and does not show the intrinsic near-infrared excess. Type 2 object has the $\mathrm{H} \alpha$ emission line with its equivalent width less than $10 \AA$ and shows the intrinsic near-infrared excess. Type 3 object has the $\mathrm{H} \alpha$ 


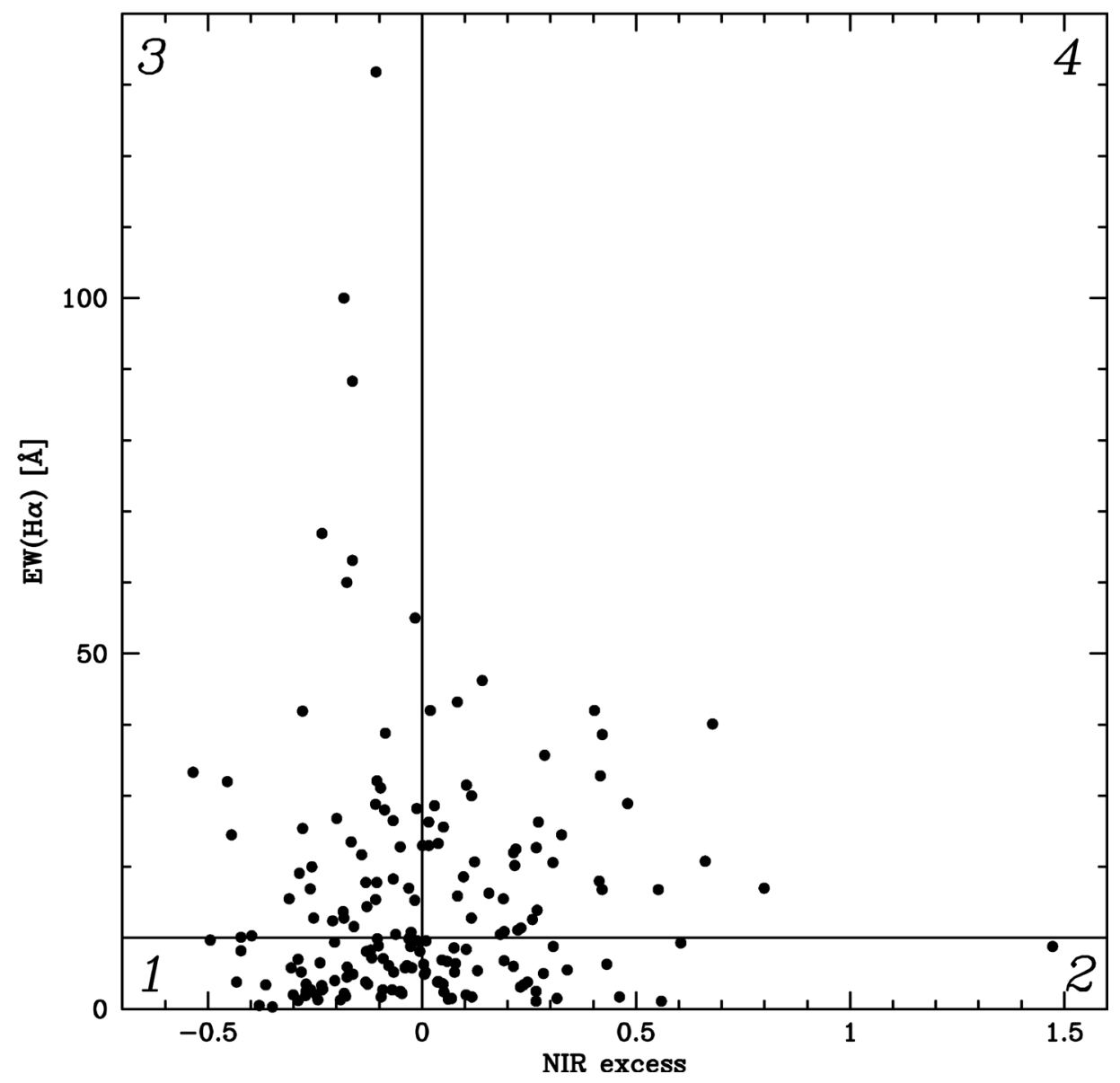

Figure 2. The intrinsic near-infrared excess and the equivalent widths of the $\mathrm{H} \alpha$ emission line of the TTSs associated with the BRCs. Type 3 objects and Type 4 objects are identified as CTTSs and Type 1 objects as WTTSs. Type 2 objects are newly identified with this study.

emission line with its equivalent width larger than $10 \AA$ and does not show the intrinsic near-infrared excess. Type 4 object has the $\mathrm{H} \alpha$ emission line with its equivalent width larger than $10 \AA$ and shows the intrinsic near-infrared excess. For the $\mathrm{H} \alpha$ emission line stars in the observed BRCs, $30 \%$ stars are classified into Type 1, 21\% into Type 2, 25\% into Type 3, and $24 \%$ into Type 4 . We do not find mass nor extinction dependencies on the object types.

$\mathrm{H} \alpha$ emission line stars in other star forming regions were also investigated. We used the $\mathrm{H} \alpha$ equivalent widths listed in [25] for the objects in the Taurus molecular cloud, [26] for the Chamaeleon molecular cloud, [27] for the $\rho$ Ophiuchi cloud, [28] and [29] for BRCs, and [30] for the Orion cluster. Near-infrared magnitudes of the $\mathrm{H} \alpha$ emission line stars were taken from 2MASS catalog. Table 3 shows the classification of the $\mathrm{H} \alpha$ emission line stars in the regions. It is revealed that Type 2 objects are abundant in the massive star forming regions such as BRCs compared to those in the low-mass star forming regions such as the Taurus molecular cloud. On the other hand, difference in the spatial distributions of Type 2 objects and the other type objects is not identified for the BRCs observed in this study and IC 1396 cluster. 
Table 3. Classification of the $\mathrm{H} \alpha$ emission line stars.

\begin{tabular}{ccccc}
\hline Region & Type 1 & Type 2 & Type 3 & Type 4 \\
\hline BRC (this study) & $52(30 \%)$ & $36(21 \%)$ & $44(25 \%)$ & $41(24 \%)$ \\
BRC (Ikeda et al.) & $177(36 \%)$ & $108(22 \%)$ & $98(20 \%)$ & $108(22 \%)$ \\
IC 1396 (Nakano et al.) & $236(37 \%)$ & $45(7 \%)$ & $249(39 \%)$ & $109(17 \%)$ \\
Orion & $167(31 \%)$ & $16(3 \%)$ & $221(41 \%)$ & $135(25 \%)$ \\
Taurus & $53(42 \%)$ & $5(4 \%)$ & $25(20 \%)$ & $42(34 \%)$ \\
Chamaeleon & $44(63 \%)$ & $3(4 \%)$ & $14(18 \%)$ & $12(15 \%)$ \\
$\rho$ Oph & $185(81 \%)$ & $7(3 \%)$ & $21(9 \%)$ & $16(7 \%)$ \\
\hline
\end{tabular}

The general concept that a low-mass star evolves from a CTTS to a WTTS is well established by many observational studies of nearby low-mass star forming regions. In our definition, Type 3 and Type 4 objects correspond to CTTSs and Type 1 objects to WTTSs. Dissipation process of a circumstellar disk around a low-mass star in strong UV field emanating from a nearby OB star has been widely discussed. [31] observed the Arches cluster near the Galactic center. They identified a significant population of near-infrared excess sources. The disk fraction of B-type star was derived as $6 \%$ in the Arches cluster. On the other hand, the fraction was as low as $3 \%$ in the vicinity of O-type stars in the cluster core. They concluded that disk dissipation process was more rapid in compact starburst clusters than in moderate star-forming environments.

Disk dissipation process due to UV radiation is also examined by numerical simulations. [32] considered circumstellar disk evolution in strong far-UV radiation fields from external stars. It is revealed that the UV radiation from nearby OB stars heats the gas near the disk edge and effectively drives mass loss from circumstellar disks. They also found that the UV radiation photoevaporates disks and disk radii are truncated to less than $\sim 100$ AU.

Type 2 objects have a weak $\mathrm{H} \alpha$ emission line but show the intrinsic near-infrared excess. Those are classified into WTTSs from optical spectroscopy, albeit into CTTSs from near-infrared photometry. The general concept of the formation process of a low-mass star does not involve such objects. We propose two hypotheses for Type 2 objects. The first hypothesis involves the idea that a Type 2 object is a WTTS with a flaring circumstellar disk. The $\mathrm{H} \alpha$ emission line stars are associated with the BRCs. Photons from the nearby OB star ionize hydrogen atoms outside the BRCs and excite hydrogen atoms at the boundary of the BRCs. Type 2 objects may be irradiated by UV photons from the nearby OB star. [33] calculated the structure of a circumstellar disk irradiated by UV radiation emanating from a nearby massive star, based on the circumstellar disk model of [34] and [35]. They indicated that the disk has large scale height, because gas in the disk is heated by the UV radiation then expands. We constructed SEDs of the $\mathrm{H} \alpha$ emission line stars in IC 1396 cluster with the photometric data of Guide Star Catalog, 2MASS catalog, and WISE catalog, then fitted them with the SED 
model of [36]. Surface height of the circumstellar disk is expressed as,

$$
H(r)=H_{0}\left(\frac{r}{R_{0}}\right)^{\beta},
$$

where $H_{0}$ is the disk half-thickness, $R_{0}$ is the radius of the central star, and $\beta$ is the flaring parameter [37]. $\beta$ is deduced to be between 1.00 and 1.20 for all objects. The average $\beta$ are $1.067 \pm 0.008,1.095 \pm 0.015$, and $1.106 \pm 0.009$ for Type 1, 3, and 4 objects, respectively, while that of Type 2 objects is as high as $1.125 \pm 0.011$. Large $\beta$ of Type 2 objects supports the idea that those are WTTSs with a highly flared disk, although the difference in $\beta$ between Type 2 and Type 4 is less than $2 \sigma$ significance level. Spatial distribution of Type 2 objects should be inhomogeneous, if this hypothesis is valid. One may guess rich population of Type 2 objects outside the BRC or at the surface of the BRC. However, we do not find such spatial distribution of Type 2 objects.

Another hypothesis is that Type 2 objects are pre-transitional disk objects. A transitional disk object has a circumstellar disk with an inner hole created by photo evaporation of a central star or planet formation. A pre-transitional disk object has a small and optically thick disk in the inner hole of the circumstellar disk. If near-infrared excess arises from the inner disk and a small amount of material accretes from the inner disk to the photosphere, then the object is classified into Type 2 object. We investigated $\mathrm{H} \alpha$ strength and near-infrared excess of five pre-transitional disk objects listed in [38]. Four objects are located in the Type 2 region, or in Type 4 region near the border between Type 2 region and Type 4 region in the near-infrared excess and $\mathrm{H} \alpha$ equivalent width diagram. Similarities between Type 2 objects and pre-transitional disk objects is also found in the near-infrared and WISE colors. We plotted the TTSs in the BRCs, the transitional disk objects in the L 1641 cloud [39], and the known pre-transitional disk objects on the near-infrared and WISE color diagram (Figure 3). Majority of Type 2 objects are not plotted in the transitional disk object region, but their $K$ - [4.6] colors are redder than that of the transitional disk objects. The known pre-transitional disk objects and Type 2 objects in the BRCs are plotted on the similar region in the diagram.

[40] carried out near-infrared polarimetry of BRC 74. They found that the magnetic field in the layer just behind the rim ran along the rim. The estimated magnetic field strength was $\sim 90 \mu \mathrm{G}$, stronger than that far inside, $\sim 30 \mu \mathrm{G}$, thereby suggesting that the magnetic field inside the rim is enhanced by the UV-radiation-induced shock. A proto-planetary disk has an inner stable region and an outer unstable region, if it magnetizes. [41] indicated that the boundary between stable and unstable regions is located at $\sim 20 \mathrm{AU}$ from the central star and the stable region shrinks in strong magnetic field environment. An abundant population of Type 2 objects in the BRCs may indicate slow evolution from CTTSs to WTTSs. Geometric structure and evolution timescale of a proto-planetary disk in the close vicinity of the central star under a strong magnetic field is to be investigated. 


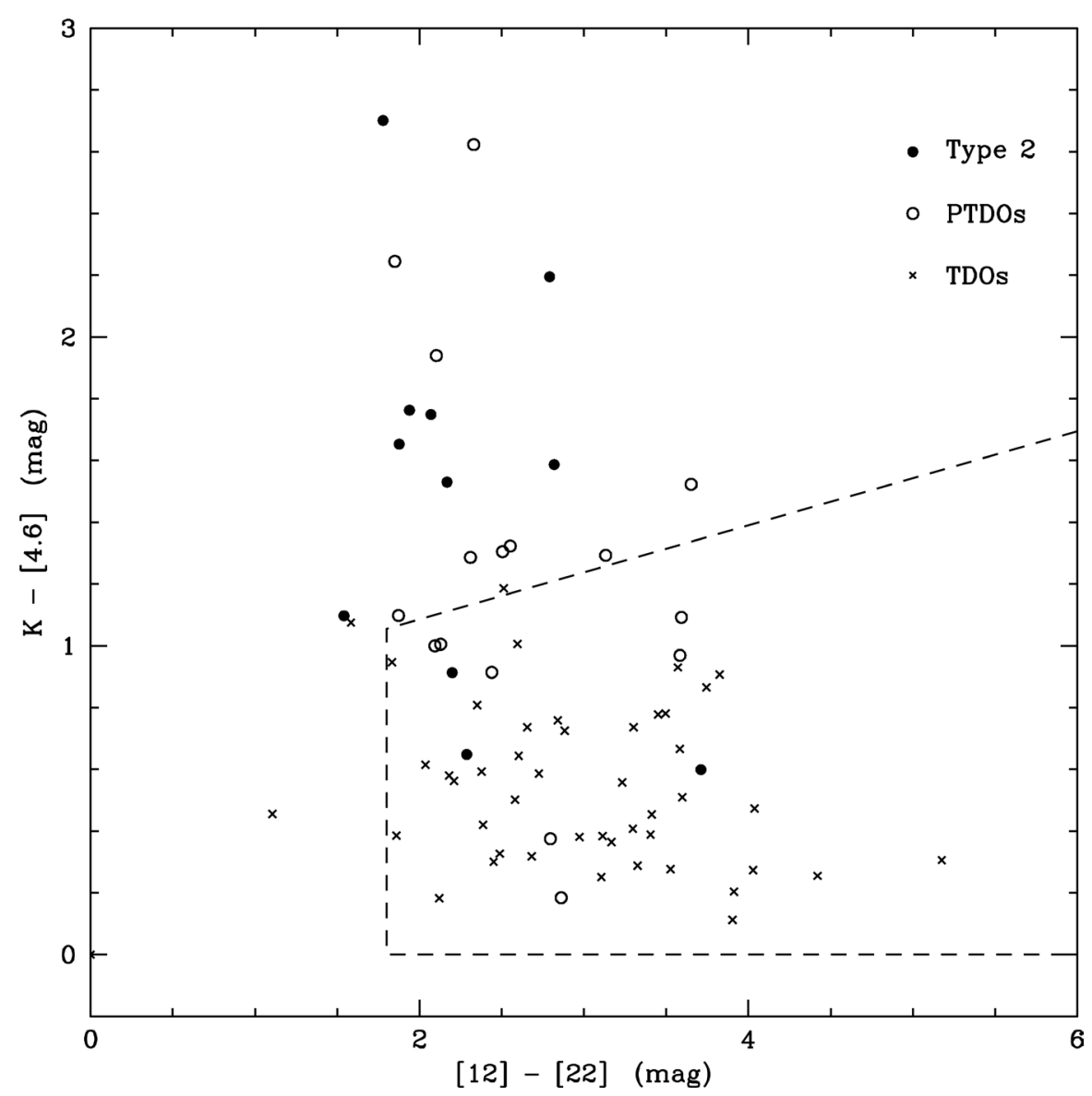

Figure 3. The near-infrared $K$-band and WISE $4.6 \mu \mathrm{m}$-, $12 \mu \mathrm{m}$-, and $22 \mu \mathrm{m}$-bands color diagram of Type 2 objects, transitional disk objects, and pre-transitional disk objects. The region enclosed by a dashed line is the region of the transitional disk objects. Type 2 objects and pre-transitional disk objects have redder color in $K$-[4.6] color.

\section{Conclusion}

We have conducted slit-less optical spectroscopy for 14 bright rimmed clouds and found $173 \mathrm{H} \alpha$ emission line stars. Among them, 36 objects have a weak $\mathrm{H} \alpha$ emission line, but show intrinsic near-infrared excess. Those are identified as WTTSs with optical spectroscopy, but as CTTSs with near-infrared photometry. The general concept of the formation process of a low-mass star does not involve such objects. Those might be weak-line $\mathrm{T}$ Tauri stars with a flared circumstellar disk in which gas is heated by ultraviolet radiation from a nearby early-type star. Alternatively, those might be pre-transitional disk objects.

\section{Acknowledgements}

We thank the telescope staff members and operators at the IUCAA 2 m Telescope. This study was partly supported by the JSPS-DST collaboration.

\section{Conflicts of Interest}

The authors declare no conflicts of interest regarding the publication of this paper. 


\section{References}

[1] Joy, A.H. (1945) T Tauri Variable Stars. The Astrophysical Journal, 102, 168-195. https://doi.org/10.1086/144749

[2] Meyer, M.R., Calvet, N. and Hillenbrand, L.A. (1997) Intrinsic Near-Infrared Excesses of T Tauri Stars: Understanding the Classical T Tauri Star Locus. The Astrophysical Journal, 114, 288-300. https://doi.org/10.1086/118474

[3] Bertout, C., Basri, G. and Bouvier, J. (1988) Accretion Disks around T Tauri Stars. The Astrophysical Journal, 330, 350-373. https://doi.org/10.1086/166476

[4] Lada, C.J. and Adams, F.C. (1992) Interpreting Infrared Color-Color Diagrams-Circumstellar Disks around Low- and Intermediate-Mass Young Stellar Objects. The Astrophysical Journal, 393, 278-288. https://doi.org/10.1086/171505

[5] Bertout, C., Siess, L. and Cabrit, S. (2007) The Evolution of Stars in the Taurus-Auriga T Association. Astronomy \& Astrophysics, 473, L21-L24. https://doi.org/10.1051/0004-6361:20078276

[6] Takagi, Y., Itoh, Y. and Oasa, Y. (2014) Disk Dissipation Timescale of Pre-Main Sequence Stars in Taurus. Publications of the Astronomical Society of Japan, 66, 88. https://doi.org/10.1093/pasj/psu062

[7] Sugitani, K., Fukui, Y. and Ogura, K. (1991) A Catalog of Bright-Rimmed Clouds with IRAS Point Sources: Candidates for Star Formation by Radiation-Driven Implosion. I. The Northern Hemisphere. The Astrophysical Journal Supplement Series, 77, 59-66. https://doi.org/10.1086/191597

[8] Sugitani, K. and Ogura, K. (1994) A Catalog of Bright-Rimmed Clouds with IRAS Point Sources: Candidates for Star Formation by Radiation-Driven Implosion. 2: The Southern Hemisphere. The Astrophysical Journal Supplement Series, 92, 163-172. https://doi.org/10.1086/191964

[9] Niwa, T., et al. (2009) Millimeter-Wave Survey of Molecular Clouds around the W5-East Triggered Star-Forming Region. Astronomy \& Astrophysics, 500, 1119-1129. https://doi.org/10.1051/0004-6361/200811065

[10] Ogura, K., et al. (2002) $\mathrm{H} \alpha$ Emission Stars and Herbig-Haro Objects in the Vicinity of Bright-Rimmed Clouds. The Astronomical Journal, 123, 2597-2626. https://doi.org/10.1086/339976

[11] Hayashi, M., Itoh, Y. and Oasa, Y. (2012) Near-Infrared Survey of Bright Rimmed Clouds. Publications of the Astronomical Society of Japan, 64, 96. https://doi.org/10.1093/pasj/64.5.96

[12] Sugitani, K., Tamura, M. and Ogura, K. (1995) Young Star Clusters in Bright-Rimmed Clouds: Small-Scale Sequential Star Formation? The Astrophysical Journal, 455, L39-L41. https://doi.org/10.1086/309808

[13] Matsuyanagi, I., et al. (2006) Sequential Formation of Low-Mass Stars in the BRC 14 Region. Publications of the Astronomical Society of Japan, 58, L29-L34. https://doi.org/10.1093/pasj/58.4.L29

[14] Siess, L., Dufour, E. and Forestini, M. (2000) An Internet Server for Pre-Main Sequence Tracks of Low- and Intermediate-Mass Stars. Astronomy \& Astrophysics, 358, 593-599.

[15] Baraffe, I., et al. (1998) Evolutionary Models for Solar Metallicity Low-Mass Stars: Mass-Magnitude Relationships and Color-Magnitude Diagrams. Astronomy \& Astrophysics, 337, 403-412.

[16] Baraffe, I., et al. (2003) Evolutionary Models for Cool Brown Dwarfs and Extrasolar Giant Planets. The Case of HD 209458. Astronomy \& Astrophysics, 402, 701-712. 
https://doi.org/10.1051/0004-6361:20030252

[17] Chauhan, N., et al. (2009) Triggered Star Formation and Evolution of T-Tauri Stars in and around Bright-Rimmed Cloud. Monthly Notices of the Royal Astronomical Society, 396, 964-983. https://doi.org/10.1111/j.1365-2966.2009.14756.x

[18] Caramazza, M., et al. (2012) Star Formation in the Outer Galaxy: Coronal Properties of NGC 1893. Astronomy \& Astrophysics, 539, A74. https://doi.org/10.1051/0004-6361/201117256

[19] Dolan, C.J. and Mathieu, R.D. (1999) A WIYN Lithium Survey for Young Stars in the $\lambda$ Orionis Star-Forming Region. The Astronomical Journal, 118, 2409-2423. https://doi.org/10.1086/301075

[20] Bell, C.P.M., et al. (2013) Pre-Main Sequence Isochrones II. Revising Star and Planet Formation Time-Scales. Monthly Notices of the Royal Astronomical Society, 434, 806-831. https://doi.org/10.1093/mnras/stt1075

[21] Maheswar, G., et al. (2007) Champagne Flow and Triggered Star Formation in NGC 1893. Monthly Notices of the Royal Astronomical Society, 379, 1237-1247. https://doi.org/10.1111/j.1365-2966.2007.12020.x

[22] Prisinzano, L., et al. (2011) Star Formation in the Outer Galaxy: Membership and Fundamental Parameters of the Young Open Cluster NGC 1893. Astronomy \& Astrophysics, 527, A77. https://doi.org/10.1051/0004-6361/201015482

[23] Wang, J., et al. (2009) A Chandra Study of the Rosette Star-Forming Complex. II. Clusters in the Rosette Molecular Cloud. The Astrophysical Journal, 696, 47-65. https://doi.org/10.1088/0004-637X/696/1/47

[24] Wang, J., et al. (2008) A Chandra Study of the Rosette Star-Forming Complex. I. The Stellar Population and Structure of the Young Open Cluster NGC 2244. The Astrophysical Journal, 675, 464-490. https://doi.org/10.1086/526406

[25] Kenyon, S.J. and Hartmann, L. (1995) Pre-Main-Sequence Evolution in the Taurus-Auriga Molecular Cloud. The Astrophysical Journal Supplement Series, 101, 117-171. https://doi.org/10.1086/192235

[26] Frasca, A., et al. (2015) The Gaia-ESO Survey: Chromospheric Emission, Accretion Properties, and Rotation in $\gamma$ Velorum and Chamaeleon I. Astronomy \& Astrophysics, 575, A4. https://doi.org/10.1051/0004-6361/201424409

[27] Erickson, K.L., et al. (2011) The Initial Mass Function and Disk Frequency of the Rho Ophiuchi Cloud: An Extinction-Limited Sample. The Astrophysical Journal, 142, A140. https://doi.org/10.1088/0004-6256/142/4/140

[28] Ikeda, H., et al. (2008) Sequential Star Formation in a Cometary Globule (BRC37) of IC1396. The Astrophysical Journal, 135, 2323-2335. https://doi.org/10.1088/0004-6256/135/6/2323

[29] Nakano, M., et al. (2012) Wide-Field Survey of Emission-Line Stars in IC 1396. The Astrophysical Journal, 143, A61. https://doi.org/10.1088/0004-6256/143/3/61

[30] Szegedi-Elek, E., et al. (2013) A New Halpha Emission-Line Survey in the Orion Nebula Cluster. The Astrophysical Journal Supplement Series, 208, A28. https://doi.org/10.1088/0067-0049/208/2/28

[31] Stolte, A., et al. (2010) Disks in the Arches Cluster-Survival in a Starburst Environment. The Astrophysical Journal, 718, 810-831. https://doi.org/10.1088/0004-637X/718/2/810

[32] Anderson, K.R., Adams, F.C. and Calvet, N. (2013) Viscous Evolution and Photoevaporation of Circumstellar Disks Due to External Far Ultraviolet Radiation Fields. The Astrophysical Journal, 774, A9. https://doi.org/10.1088/0004-637X/774/1/9 
[33] Walsh, C., et al. (2014) Complex Organic Molecules in Protoplanetary Disks. Astronomy \& Astrophysics, 563, A33. https://doi.org/10.1051/0004-6361/201322446

[34] Nomura, H. and Millar, T.J. (2005) Molecular Hydrogen Emission from Protoplanetary Disks. Astronomy \& Astrophysics, 438, 923-938. https://doi.org/10.1051/0004-6361:20052809

[35] Nomura, H. and Nakagawa, Y. (2006) Dust Size Growth and Settling in a Protoplanetary Disk. The Astrophysical Journal, 640, 1099-1109. https://doi.org/10.1086/500251

[36] Robitaille, T.P., et al. (2006) Interpreting Spectral Energy Distributions from Young Stellar Objects. I. A Grid of 200,000 YSO Model SEDs. The Astrophysical Journal Supplement Series, 167, 256-285. https://doi.org/10.1086/508424

[37] Kenyon, S.J. and Hartmann, L. (1987) Spectral Energy Distributions of T Tauri Stars-Disk Flaring and Limits on Accretion. The Astrophysical Journal, 323, 714-733. https://doi.org/10.1086/165866

[38] van der Marel, N., et al. (2016) The (w)hole Survey: An Unbiased Sample Study of Transition Disk Candidates Based on Spitzer Catalogs. Astronomy \& Astrophysics, 592, A126. https://doi.org/10.1051/0004-6361/201628075

[39] Fang, M., et al. (2013) Young Stellar Objects in Lynds 1641: Disks, Accretion, and Star Formation History. The Astrophysical Journal Supplement Series, 207, A5. https://doi.org/10.1088/0067-0049/207/1/5

[40] Kusune, T., et al. (2015) Near-IR Imaging Polarimetry toward a Bright-Rimmed Cloud: Magnetic Field in SFO 74. The Astrophysical Journal, 798, A60. https://doi.org/10.1088/0004-637X/798/1/60

[41] Sano, T., et al. (2000) Magnetorotational Instability in Protoplanetary Disks. II. Ionization State and Unstable Regions. The Astrophysical Journal, 543, 486-501. https://doi.org/10.1086/317075 